\title{
Lagrangian relaxation of the generic materials and operations planning model
}

Gregorio Rius Sorolla, Julien Maheut, Jairo Rafael Coronado Hernandez, Jose P. Garcia Sabater

\begin{abstract}
The supply chain management requires increasingly proposals for the production programming planning that brings together its special singularities. Solving coexisting products and alternative processes or by-products must be allowed by the mathematical programming models. The generic materials and operations planning (GMOP) formulation allows operating with different materials and process lists. The paper presents a procedure to solve the versatile GMOP model by the Lagrange Relaxation. The subgradient update method of the lagrangian multiplier is compared with a linear update method. Obtaining lower bound faster compared to the linear method is allowed by the subgradient method, but the linear method provides better solutions after certain iterations.
\end{abstract}

\section{Keywords}

GMOP, Lagrangian relaxation, Subgradient 